\title{
Sick euthyroid syndrome is associated with poor prognosis in patients with ST segment elevation myocardial infarction undergoing primary percutaneous intervention
}

\author{
Kazım Serhan Özcan ${ }^{1}$, Damirbek Osmonov ${ }^{2}$, Ercan Toprak ${ }^{3}$, Barış Güngör ${ }^{3}$, \\ Adem Tatlısu ${ }^{3}$, Ahmet Ekmekçi ${ }^{3}$, Adnan Kaya ${ }^{3}$, Gülşah Tayyareci ${ }^{3}$, İzzet Erdinler ${ }^{3}$ \\ ${ }^{1}$ Department of Cardiology, Derince Training and Research Hospital, Kocaeli, Turkey \\ ${ }^{2}$ Department of Cardiology, Almaty SEMA Hospital, Almaty, Kazakhstan \\ ${ }^{3}$ Department of Cardiology, Siyami Ersek Cardiovascular and Thoracic Surgery Center, Istanbul, Turkey
}

\begin{abstract}
Background: Concomitant thyroid and heart disease are frequently encountered in clinical practice. There are many studies evaluating thyroid function in acute and critical conditions. Information on thyroid dysfunction in ST-segment elevation myocardial infarction (STEMI) is limited; its correlation with short and long-term outcome is not fully known.

Methods: Four hundred and fifty seven patients diagnosed with STEMI in our emergency department were included in the study. Patients were divided into two groups: patients with normal thyroid function (euthyroid) and patients with thyroid dysfunction. STEMI was diagnosed with 12 derivation surface electrocardiogram. Thyroid hormone levels (TSH, free T3 and free T4) were measured. Patients with other acute coronary syndromes and endocrine pathologies except diabetes mellitus were excluded. Two patient groups were compared in terms of in-hospital and long-term outcome.
\end{abstract}

Results: Out of 457, 72 (15\%) patients with thyroid dysfunction were detected. The other patients were euthyroid and constituted the control group. In-hospital cardiogenic shock $(15 \% \mathrm{vs}$. $3 \%$ in the control group; $p<0.01)$ and death (7\% vs. $1 \%$ in the control group; $p<0.01)$ were more frequently observed in the thyroid dysfunction group. In the subgroup analysis, it was observed that patients with sick euthyroid syndrome have the poorest outcome. Other markers for poor outcome were anemia and renal failure.

Conclusions: Thyroid dysfunction, particularly sick euthyroid syndrome, was found to be related to in-hospital and long term mortality in patients with STEMI undergoing primary percutaneous intervention. (Cardiol J 2014; 21, 3: 238-244)

Key words: thyroid dysfunction, sick euthyroid syndrome, ST segment elevation myocardial infarction

Address for correspondence: Damirbek Osmonov, MD, Department of Cardiology, Almaty SEMA Hospital, Nauryzbai batyr str. 31, 050000 Almaty, Kazakhstan, tel: + 7727 3996666, fax: + 7727 2780066, e-mail: damirbeko@yahoo.com Received: 01.05.2013 Accepted: 19.06.2013 


\section{Introduction}

There is a close relationship between thyroid hormone levels and cardiovascular diseases [1]. Thyroid hormones critically regulate cardiac function through several genes encoding important structural and functional proteins in the myocardium [2]. Both hypothyroidism and hyperthyroidism have been associated with increased risk of coronary artery disease in previous reports [3]. Thyroid hormones also have been implicated in the mortality of other cardiac diseases like heart failure, arterial hypertension, atherosclerosis and dyslipidemia [4-8].

There are many studies evaluating thyroid function in acute and critical conditions [9]. Information on thyroid dysfunction in ST-segment elevation myocardial infarction (STEMI) is limited; its correlation with short and long-term outcome is not fully known. The aim of our study was to investigate the association between thyroid dysfunction and prognosis of STEMI patients who underwent primary coronary intervention.

\section{Methods}

\section{Patient population}

Our study was conducted between June 2010 and June 2012 in Siyami Ersek Thoracic and Cardiovascular Surgery Hospital, a tertiary reference center. We prospectively enrolled consecutive 457 patients who received acute STEMI diagnosis in the emergency department of our hospital. Patients with other types of acute coronary syndrome, chronic liver disease, kidney disease requiring dialysis, malignancy, active systemic infection, endocrine disease other than diabetes mellitus were excluded.

Informed consents of patients were obtained before the study. The study was authorized by the institutional ethics committee.

\section{Data sources}

Demographic data and clinical history concerning age, sex, diabetes mellitus, hyperlipidemia, smoking, family history of coronary artery disease, earlier drug use were obtained from all patients. Electrocardiogram (ECG) was obtained from all patients after first medical contact for diagnosis. Blood pressures of all patients were measured at admission. Physical examination was also performed. All patients underwent coronary catheterization for primary angioplasty after receiving aspirin (300 mg) and clopidogrel (300 mg loading dose). Angiographic data of the patients were collected from the cardiac catheterization laboratory records. Emergency cardiac catheterization was performed using percutaneous femoral approach using nonionic iso-osmolar contrast media. A coronary artery stenosis more than $50 \%$ was considered clinically significant. Occlusion of the infarct related artery (IRA) was crossed by using a 0.014-inch guidewire. Primary angioplasty including balloon angioplasty and/or stent implantation was performed only for IRA based on the lesion anatomy.

\section{Definitions}

Patients who were admitted to emergency department of our hospital with typical ischemic symptoms (chest pain lasting for $>30 \mathrm{~min}$ ) were diagnosed using 12 derivation surface ECG. When indicated, right (V3R-V4R) and posterior (V7-V9) derivations were also obtained. The ECG criteria for STEMI are ST-segment elevation of $\geq 2 \mathrm{~mm}$ in at least two contiguous leads or new onset of complete left bundle branch block.

Thyroid hormone levels of all patients; free $\mathrm{T} 3$, free T4 and TSH, were measured in the laboratory of our hospital. The reference values of our laboratory are $0.34-5.0 \mu \mathrm{IU} / \mathrm{mL}$ for TSH; 0.59 $-1.3 \mathrm{ng} / \mathrm{dL}$ for free T4; $2.3-4.0 \mathrm{pg} / \mathrm{mL}$ for free T3.

Patients with normal TSH and free T4 levels, and decreased free T3 levels were defined as sick euthyroid syndrome; increased TSH levels, normal free T3 and T4 levels as subclinical hypothyroidism; increased TSH levels, decreased free T3 and T4 levels as overt hypothyroidism; decreased TSH levels, normal free T3 and T4 levels as subclinical hyperthyroidism; decreased TSH levels, increased free T3 and T4 levels as overt hyperthyroidism.

Subjects with thyroid dysfunction were compared to subjects with normal thyroid function. As subjects with overt hypothyroidism $(n=2)$ and overt hyperthyroidism $(\mathrm{n}=2)$ were very few, thyroid dysfunction patients were only subgrouped as hypothyroidism, hyperthyroidism and sick euthyroid syndrome.

\section{Collection of serum samples}

Venous blood samples were collected from all patients within the first $12 \mathrm{~h}$ of admission for laboratory analysis.

\section{Biochemical tests}

Hemoglobin, hematocrit and platelet counts, blood glucose, creatinine, sodium, potassium, AST, ALT and CK-MB levels of all patients were measured at admission. Fasting lipid (triglycerides, LDL-cholesterol, HDL-cholesterol) and thyroid hormone levels were all measured in the laboratory of our hospital as well. 


\section{Follow-up}

All patients were monitored for in-hospital major adverse cardiac event (MACE) throughout hospitalization. In-hospital MACE refers to ventricular arrhythmias, recurrent infarct, target vessel revascularization (TVR), cardiogenic shock and death. Follow-up data were obtained from hospital records or by interviewing the patients (directly or by telephone), their families and personal physicians. Mean follow-up duration after discharge was $14.4 \pm 5.4$ months.

\section{Statistical analysis}

All data were presented as mean \pm standard deviation for parametric variables and as percentages for categorical variables unless stated otherwise. Continuous variables were checked for the normal distribution assumption using the Kolmogorov-Smirnov statistics. Differences between patients and control subjects were evaluated using the 2 -sample $t$ test and Mann-Whitney $\mathrm{U}$ test as appropriate. Categorical variables were tested by Pearson's $\chi^{2}$ test and Fisher's Exact Test. For clinical outcomes, Kaplan-Meier estimates and curves were generated, and comparisons were made using log-rank test. Forward stepwise multivariate logistic regression models were created to identify the independent predictors of in-hospital and long term adverse events. Variables with a $\mathrm{p}$ value $<0.10$ in univariate analysis were included in the multivariate model. The predictive value of thyroid function on clinical outcomes was examined using plasma levels of TSH, fT3 and freeT4 as continuous variables. In addition, we performed regression analysis using hypothyroidism, hyperthyroidism, sick euthyroid syndrome and control group as binary variables. All statistical studies were carried out using Statistical Package for Social Sciences software (SPSS 16.0 for Windows, SPSS Inc., Chicago, Illinois) and a $\mathrm{p}<0.05$ was considered statistically significant.

\section{Results}

A total number of 457 patients with STEMI who were treated with primary percutaneous coronary intervention (PCI) were prospectively included in our study. Seventy two patients (57 male; mean age $60.01 \pm 13.87$ years) had thyroid dysfunction and 385 patients were with normal thyroid function (324 male; mean age $55.56 \pm 12.29$ years) which constituted the control group. Demographic and clinical properties of subjects with thyroid dysfunction and controls were depicted in Table 1.
Patients with thyroid dysfunction were older $(p<0.01)$, had lower free T3, hemoglobin and higher creatinine levels. The other clinical, angiographic and laboratory parameters including plasma TSH and free T4 levels were similar between the two groups. Thyroid dysfunction group included 17 subjects with hypothyroidism, 25 subjects with hyperthyroidism and 30 subjects with sick euthyroid syndrome. Seven of the patients with thyroid dysfunction had prior diagnosis of hypothyroidism and only 1 subject had prior diagnosis of hyperthyroidism.

In-hospital and long term clinical outcomes were listed in Table 2. Five $(6.9 \%)$ patients with thyroid dysfunction and $5(1.2 \%)$ patients with normal thyroid function died during hospitalization period. The incidence of MACE was significantly higher in patients with thyroid dysfunction $(15.2 \%$ vs. $7.8 \%, p=0.04$ ) and was primarily driven by in-hospital mortality.

The mean follow-up period of participants was $14.4 \pm 5.4$ months. During this period, $19(29.2 \%)$ MACE occurred in patients with thyroid dysfunction and $61(16.1 \%)$ MACE occurred in control group which was statistically significant $(\mathrm{p}=0.01)$. The incidence of mortality and TVR was significantly higher in patients with thyroid dysfunction $(\mathrm{p}<0.01$, $\mathrm{p}=0.04$, respectively). In subgroup analysis, the incidence of in-hospital and long term mortality ( $\mathrm{n}=3 / 30$ and $4 / 27$ respectively) was highest in subjects with sick euthyroid syndrome.

Tables 3 and 4 list the independent predictors of in-hospital and long term MACE in study population. Correlation of thyroid function with clinical outcomes was analyzed separately as continuous variables and subgroups of thyroid function namely control, hypothyroidism, hyperthyroidism and sick euthyroid syndrome. In univariate analysis, age, left ventricular ejection fraction, admission glucose levels, peak CK-MB levels and presence of sick euthyroid syndrome were associated with in-hospital MACE. Plasma levels of free T3 were weakly associated with in-hospital MACE only in univariate analysis but not in multivariate analysis. In multivariate analysis, increased age (HR 1.03, 95\% CI 1.01-1.06, $\mathrm{p}=0.02$ ) and presence of sick euthyroid syndrome (HR 3.30, 95\% CI 1.22-8.94, $\mathrm{p}=0.02$ ) were the only independent predictors of in-hospital MACE.

Univariate logistic regression analysis revealed age, female gender, admission hemoglobin, creatinine, free T3 levels, peak CK-MB levels and presence of sick euthyroid syndrome as predictors of long term MACE with $\mathrm{p}$ values lower than 0.10 . 
Table 1. Baseline characteristics of the study population.

\begin{tabular}{|c|c|c|c|}
\hline & Thyroid dysfunction ( $n=72$ ) & Control $(\mathrm{n}=385)$ & $\mathbf{P}$ \\
\hline Age & $60.01 \pm 13.87$ & $55.56 \pm 12.29$ & $<0.01$ \\
\hline Male gender & $57(79.1 \%)$ & $324(84.1 \%)$ & 0.30 \\
\hline Hypertension & $37(51.3 \%)$ & $166(43.1 \%)$ & 0.19 \\
\hline Diabetes mellitus & $15(20.8 \%)$ & $90(23.3 \%)$ & 0.63 \\
\hline Hyperlipidemia & $17(23.6 \%)$ & $76(19.7 \%)$ & 0.45 \\
\hline Smoking & $48(66.6 \%)$ & $281(72.9 \%)$ & 0.27 \\
\hline Family history for CAD & $19(26.4 \%)$ & $122(31.6 \%)$ & 0.57 \\
\hline \multicolumn{4}{|l|}{ Thyroid function: } \\
\hline Hypothyroidism & $17(23.6 \%)$ & - & \\
\hline Hyperthyroidism & $25(34.7 \%)$ & - & \\
\hline Sick euthyroid syndrome & $30(41.6 \%)$ & - & \\
\hline Systolic BP [mm Hg] & $122.80 \pm 34.93$ & $124.43 \pm 23.38$ & 0.70 \\
\hline Diastolic BP [mm Hg] & $73.73 \pm 18.84$ & $77.20 \pm 52.98$ & 0.68 \\
\hline Anterior MI & 27 & 167 & 0.35 \\
\hline Pain-to-balloon time & 5.29 & 6.20 & 0.24 \\
\hline Multivessel disease & $43(59.7 \%)$ & $211(54.8 \%)$ & 0.38 \\
\hline \multicolumn{4}{|l|}{ Laboratory parameters: } \\
\hline Hemoglobin & $13.05 \pm 1.61$ & $14.04 \pm 1.73$ & $<0.01$ \\
\hline Glucose & $162.02 \pm 69.15$ & $160.52 \pm 78.80$ & 0.88 \\
\hline Creatinine & $1.06 \pm 045$ & $0.91 \pm 0.34$ & $<0.01$ \\
\hline Trygliceride & $142.19 \pm 74.49$ & $162.76 \pm 108.26$ & 0.12 \\
\hline Total cholesterol & $181.55 \pm 53.96$ & $189.35 \pm 42.88$ & 0.22 \\
\hline LDL cholesterol & $116.84 \pm 40.22$ & $119.89 \pm 34.58$ & 0.50 \\
\hline HDL cholesterol & $38.74 \pm 11.44$ & $37.29 \pm 10.49$ & 0.30 \\
\hline Peak CK-MB & $165.54 \pm 143.97$ & $165.99 \pm 139.06$ & 0.98 \\
\hline TSH & $2.15 \pm 3.01$ & $1.64 \pm 0.95$ & 0.15 \\
\hline fT3 & $2.46 \pm 0.80$ & $2.90 \pm 0.42$ & $<0.01$ \\
\hline fT4 & $1.20 \pm 0.42$ & $1.12 \pm 0.28$ & 0.14 \\
\hline
\end{tabular}

CAD — coronary artery disease; BP — blood pressure; MI - myocardial infarction

Table 2. Comparison of in-hospital and long term major adverse cardiovascular events (MACE) in patients with thyroid dysfunction and control subjects.

\begin{tabular}{lccc}
\hline & Thyroid dysfunction $\mathbf{( n = 7 2 )}$ & Control $\mathbf{n}=\mathbf{3 8 5})$ & $\mathbf{P}$ \\
\hline In-hospital MACE: & $11 / 72$ & $30 / 385$ & 0.04 \\
Recurrent myocardial infarction & $5 / 72$ & $26 / 385$ & 0.90 \\
Target vessel revascularization & $6 / 72$ & $24 / 385$ & 0.68 \\
Mortality & $5 / 72$ & $5 / 385$ & $<0.01$ \\
Long term MACE: & $19 / 65$ & $61 / 380$ & 0.01 \\
Recurrent myocardial infarction & $11 / 65$ & $47 / 380$ & 0.34 \\
Target vessel revascularization & $12 / 65$ & $37 / 380$ & 0.04 \\
Mortality & $6 / 65$ & $8 / 380$ & $<0.01$ \\
\hline
\end{tabular}

However, in multivariate analysis increased age and presence of sick euthyroid syndrome (HR $2.83,95 \%$ CI $1.16-6.88, \mathrm{p}=0.02$ ) were the only independent predictors. The Kaplan-Meier estimate of long term survival free from MACE was significantly worse in patients with sick euthyroid syndrome (Fig. 1).

\section{Discussion}

Thyroid hormones have important effects on the cardiovascular system through direct and indirect mechanisms [1]. Although STEMI and thyroid disorders are not directly related, the relationship between atherosclerosis and hypothyroidism as 
Table 3. Effects of multiple variables on the in-hospital major adverse cardiac event in univariate and multivariate logistic regression analyses.

\begin{tabular}{|c|c|c|c|c|}
\hline Variables & $\begin{array}{l}\text { Unadjusted odds } \\
\text { ratio }(95 \% \mathrm{Cl})\end{array}$ & $\mathbf{P}$ & $\begin{array}{c}\text { Adjusted odds ratio* } \\
(95 \% \mathrm{Cl})\end{array}$ & $\mathbf{P}$ \\
\hline Age & $1.04(1.01-1.065)$ & $<0.01$ & $1.03(1.01-1.06)$ & 0.02 \\
\hline Male gender & $0.68(0.31-1.48)$ & 0.32 & & \\
\hline Hypertension & $1.52(0.79-2.89)$ & 0.21 & & \\
\hline Diabetes mellitus & $1.84(0.82-3.65)$ & 0.11 & & \\
\hline Smoking & $1.43(0.66-3.09)$ & 0.36 & & \\
\hline Anterior myocardial infarction & $0.85(0.44-1.63)$ & 0.63 & & \\
\hline Pain-to-balloon time & $0.99(0.98-1.01)$ & 0.66 & & \\
\hline No-reflow & $0.62(0.19-2.07)$ & 0.44 & & \\
\hline Multivessel disease & $1.26(0.65-2.43)$ & 0.49 & & \\
\hline Left ventricular ejection fraction & $0.96(0.93-1.01)$ & 0.05 & $0.98(0.94-1.02)$ & 0.40 \\
\hline Hemoglobin & $0.89(0.75-1.07)$ & 0.22 & & \\
\hline Glucose & 1.004 (1.001-1.007) & 0.04 & $1.004(1.0-1.008)$ & 0.08 \\
\hline Creatinine & $1.60(0.76-3.38)$ & 0.21 & & \\
\hline Peak CK-MB & $1.002(1.00-1.004)$ & 0.02 & $1.001(0.99-1.004)$ & 0.23 \\
\hline TSH & $0.87(0.65-1.14)$ & 0.31 & & \\
\hline $\mathrm{fT}^{* *}$ & $0.60(0.33-1.10)$ & 0.09 & $0.75(0.37-1.51)$ & 0.42 \\
\hline fT4 & $1.91(0.81-4.46)$ & 0.14 & & \\
\hline Hypothyroidism** & $0.78(0.11-6.16)$ & 0.81 & $1.07(0.13-8.84)$ & 0.94 \\
\hline Hyperthyroidism** & $1.61(0.45-5.68)$ & 0.46 & $1.61(0.43-6.03)$ & 0.47 \\
\hline Sick euthyroid syndrome** & $3.59(1.42-9.05)$ & $<0.01$ & $3.30(1.22-8.94)$ & 0.02 \\
\hline
\end{tabular}

*Adjusted for age, left ventricular ejection fraction, plasma glucose and peak CK-MB levels; **fT3 and subgroups of thyroid dysfunction were analyzed separately

Table 4. Effects of multiple variables on long term major adverse cardiac event in univariate and multivariate logistic regression analyses.

\begin{tabular}{|c|c|c|c|c|}
\hline Variables & $\begin{array}{l}\text { Unadjusted odds } \\
\text { ratio }(95 \% \mathrm{Cl})\end{array}$ & $\mathbf{P}$ & $\begin{array}{c}\text { Adjusted odds ratio* } \\
\text { (95\% CI) }\end{array}$ & $\mathbf{P}$ \\
\hline Age & $1.03(1.01-1.05)$ & $<0.01$ & $1.03(1.01-1.05)$ & 0.02 \\
\hline Male gender & $0.49(0.28-0.89)$ & 0.02 & $0.64(0.31-1.31)$ & 0.22 \\
\hline Hypertension & $1.24(0.77-2.02)$ & 0.38 & & \\
\hline Diabetes mellitus & $1.13(0.64-1.98)$ & 0.66 & & \\
\hline Smoking & $0.81(0.49-1.39)$ & 0.46 & & \\
\hline Anterior myocardial infarction & $1.03(0.66-1.67)$ & 0.90 & & \\
\hline Pain-to-balloon time & $1.00(0.99-1.01)$ & 0.73 & & \\
\hline No-reflow & $0.87(0.39-1.94)$ & 0.74 & & \\
\hline Multivessel disease & $0.72(0.44-1.18)$ & 0.19 & & \\
\hline Left ventricular ejection fraction & $0.98(0.96-1.01)$ & 0.31 & & \\
\hline Hemoglobin & $0.86(0.75-0.98)$ & 0.03 & $1.02(0.86-1.21)$ & 0.84 \\
\hline Glucose & $1.00(0.99-1.003)$ & 0.95 & & \\
\hline Creatinine & $1.77(0.98-3.17)$ & 0.06 & $1.37(0.73-2.59)$ & 0.32 \\
\hline Peak CK-MB & $1.002(1.00-1.003)$ & 0.04 & $1.002(1.0-1.003)$ & 0.07 \\
\hline TSH & $0.90(0.75-1.08)$ & 0.28 & & \\
\hline $\mathrm{fT}^{* *}$ & $0.63(0.39-1.02)$ & 0.06 & $0.91(0.53-1.56)$ & 0.79 \\
\hline fT4 & $1.26(0.61-2.64)$ & 0.53 & & \\
\hline Hypothyroidism** & $0.77(0.18-3.64)$ & 0.77 & $0.78(0.16-3.66)$ & 0.75 \\
\hline Hyperthyroidism** & $1.73(0.66-4.55)$ & 0.26 & $1.79(0.66-4.85)$ & 0.25 \\
\hline Sick euthyroid syndrome** & $3.58(1.58-8.09)$ & $<0.01$ & $2.83(1.16-6.88)$ & 0.02 \\
\hline
\end{tabular}

${ }^{*}$ Adjusted for age, gender, left ventricular ejection fraction, plasma hemoglobin, creatinine and peak CK-MB levels; **fT3 and subgroups of thyroid dysfunction were analyzed separately 


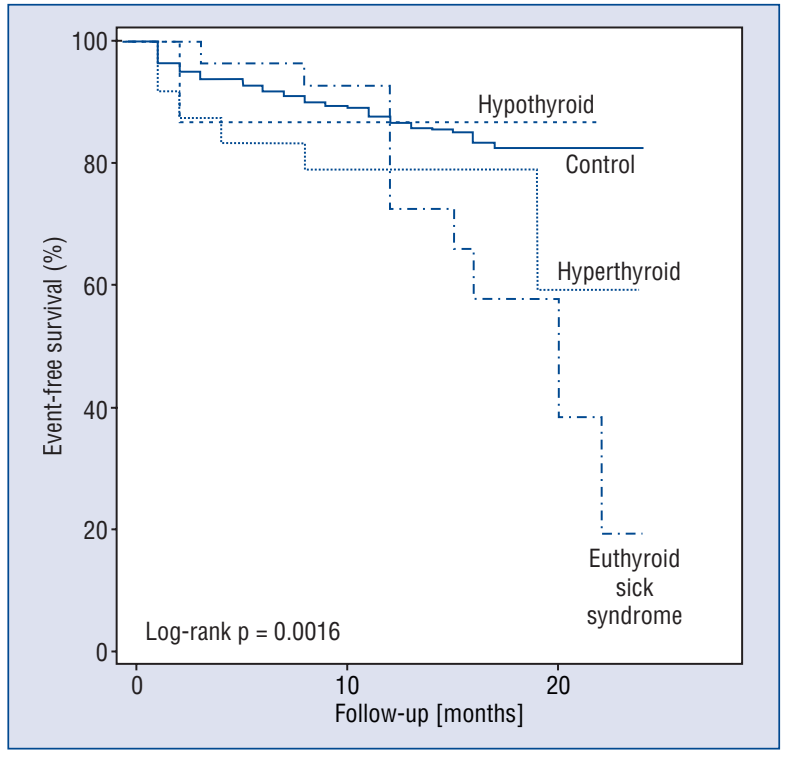

Figure 1. Kaplan-Meier estimates of survival free of major adverse cardiac event (death, myocardial infarction, and target lesion revascularization) among thyroid function subgroups.

well as cardiac arrhythmias and hyperthyroidism are well known $[1,3,7]$.

The main finding of our study was that both in-hospital and long term mortality were higher in the study group. Subgroup analysis revealed that these results were mainly driven by the high rates of mortality in patients with sick euthyroid syndrome.

Sick euthyroid syndrome is frequently observed in chronic heart failure, acute myocardial infarction (AMI) and following cardiac surgery. It is related to increased mortality in both heart failure and AMI [10-14].

Similar results were found in previous studies. In the study conducted by Pimentel et al. [15] which included 70 patients with both STEMI and non-STEMI, in-hospital mortality of the sick euthyroid group was higher. Compared to our study, sample size was small and results cover only short term mortality.

Thyroid hormone, apart from its "classical" actions on cardiac contractility and heart rhythm, appears to regulate various intracellular signaling pathways related to response to stress and cardiac remodeling. It affects cardiac remodeling by limiting reperfusion injury, and, at later states, by inducing distinct changes in cardiac chamber geometry in a time-dependent manner $[16,17]$.
Friberg et al. [18] conducted a study measuring reverse T3 levels of 331 patients with myocardial infarction. Increased reverse T3 levels were found to be related to increased 1-year mortality. The increase in reverse T3 signifies decreased free T3, which is the underlying cause of sick euthyroid syndrome. This parameter, which was not measured in our study, is a direct indicator of sick euthyroid syndrome. However, in terms of long term results, the study correlates with our findings.

The important role of thyroid hormone in the regulation of cardiac vasculature is further confirmed by the impaired coronary artery contraction, the reduced density of small arterioles, and the altered echocardiographic parameters reported in low thyroid function [19].

Consistent with our results, in a study conducted by Lazzeri et al. [20] on 641 STEMI patients, the in-hospital mortality of the sick euthyroid group was higher. The patients in this study were treated with PCI. The failure of intervention was also higher in patients with sick euthyroid syndrome.

Molinaro et al. [21] followed 1026 patients with acute cardiac diseases for 30 months after diagnosis. Cardiac mortality was found to be higher in the group with subclinical hypothyroidism and sick euthyroid syndrome. Our findings support the results of prior studies and indicate the prognostic implications of thyroid hormone levels on long-term adverse cardiac events.

In conclusion, a low free T3 level, a common phenomenon in patients with AMI, is a strong predictor of short-term and long-term poor prognoses in STEMI patients treated with primary PCI. The techniques used in detecting hormone levels are highly sensitive and available almost everywhere in modern era. Therefore, they can easily be used for predicting the in-hospital and long term prognosis in a high-mortality patient group.

\section{Limitations of the study}

This is a medium sized study covering both short and long term results. Reverse T3 levels were not measured along with other parameters. The results cannot be generalized to other patient groups because it only comprises patients diagnosed with STEMI.

\section{Conclusions}

STEMI is a frequently encountered diagnosis and still an important cause of mortality. Various scoring systems are proposed to predict short and 
long term prognoses. These scoring systems have limitations and do not include every parameter. In our study, thyroid dysfunction and especially sick euthyroid syndrome were found to be related to adverse outcomes. These results warrant further study.

\section{Conflict of interest: none declared}

\section{References}

1. Cini G, Carpi A, Mechanick J et al. Thyroid hormones and the cardiovascular system: Pathophysiology and interventions. Biomed Pharmacother, 2009; 63: 742-753.

2. Rybin V, Steinberg SF. Thyroid hormone represses protein kinase C isoform expression and activity in rat cardiac myocytes. Circ Res, 1996; 79: 388-398.

3. Asvold BO, Bjøro T, Platou C, Vatten LJ. Thyroid function and the risk of coronary heart disease: 12-year follow-up of the HUNT Study in Norway. Clin Endocrinol, 2012; 77: 911-917.

4. Hamilton MA, Stevenson LW, Luu M, Walden JA. Altered hormone metabolism in advanced heart failure. J Am Coll Cardiol, 1990; 16: 91-95.

5. Moruzzi P, Doria E, Agostoni PG. Medium-term effectiveness of Lthyroxine treament in idiopatic dilated cardiomyophathy. Am J Med, 1996; 101: 461-467.

6. Bilezikian JP, Loeb JN. The influence of hyperthyroid and hipothyroid on alpha- and beta-adrenergic receptor systems and adrenergic responsiveness. Endocr Rev, 1983; 252: H283-H290.

7. Klein I. Thyroid hormone and the cardiovascular system. Am J Med, 1990; 88: 631-637.

8. Harvey CB, Williams GR. Mechanism of thyroid hormone action. Thyroid, 2002; 12: 441-446.

9. Adler SM, Wartofsky L. The nonthyroidal illness syndrome. Endocrinol Metab Clin North Am, 2007; 36: 657-672.
10. Pingitore A, Landi P, Taddei MC, Ripoli A, Labbate A, Iervasi G. Triiodothyronine levels for risk stratification of patients with chronic heart failure. Am J Med, 2005; 118: 132-136.

11. Eber B, Schumacher M, Langsteger W et al. Changes in thyroid hormone parameters after acute myocardial infarction. Cardiology, 1995; 86: 152-156.

12. Franklyn JA, Gammage MD, Ramsden DB, Sheppard MC. Thyroid status in patients after myocardial infarction. Clin Scien, 1984; 67: 585-590.

13. Friberg L, Werner S, Eggertsen G, Ahnve S. Rapid down-regulation of thyroid hormones in acute myocardial infarction:is it cardioprotective in patients with angina? Arch Int Med, 2002; 162: 1388-1394.

14. Cerillo AG, Storti S, Clerico A, Iervasi G. Thyroid function and cardiac surgery: What should we measure and when? Ann Thoracic Surg, 2010; 89: 1010-1011.

15. Pimentel RC, Cardoso GP, Escosteguy CC, Abreu LM. Thyroid hormone profile in acute coronary syndromes. Arq Bras Cardiol, 2006; 87: 688-694.

16. Pantos C, Mourouzis I, Cokkinos DV. Thyroid hormone and cardiac repair/regeneration: From Prometheus myth to reality? Can J Physiol Pharmacol, 2012; 90: 977-987.

17. Pantos C, Mourouzis I, Cokkinos DV. New insights into the role of thyroid hormone in cardiac remodeling: Time to reconsider? Heart Fail Rev, 2011; 16: 79-96

18. Friberg L, Drvota V, Eqqersten G, Ahnve S. Association between increased levels of reverse triiodothyronine and mortality after myocardial infarction. Am J Med, 2001; 111: 699-703.

19. Liu Y, Sherer BA, Redetzke RA, Gerdes AM. Regulation of arteriolar density in adult myocardium during low thyroid conditions. Vascular Pharmacol, 2010; 52: 146-150.

20. Lazzeri C, Sori A, Picriello C, Chiostri M, Gensini GF, Valente S. Nonthyroidal illness syndrome in ST elevation myocardial infarction treated with mechanical revascularization. Int J Cardiol, 2012; 158: 103-104.

21. Molinaro S, Iervasi G, Lorenzoni V et al. Persistence of mortality risk in patients with acute cardiac diseases and mild thyroid dysfunction. Am J Med Sci, 2012; 343: 65-70. 